\title{
EDITORIAL
}

\section{To screen or not to screen mothers of preterm infants for extended-spectrum beta-lactamase-producing Enterobacteriaceae (ESBL-E)}

\section{Journal of Perinatology (2015) 35, 893-894; doi:10.1038/jp.2015.100}

Neonatal sepsis represents one of the most frequent complications for newborns hospitalized in a neonatal intensive care unit (NICU). ${ }^{1}$ In addition to the high incidence of neonatal sepsis, it is associated with increased mortality. ${ }^{2}$ Interestingly, neonates suffering from sepsis with Gram-negative bacteria are at a higher risk of death compared with Gram-positive. ${ }^{3}$ The number of multidrug-resistant Gram-negative bacteria, including extended-spectrum $\beta$-lactamase $(\mathrm{ESBL})$-producing Enterobacteriaceae (ESBL-E), is increasing worldwide not only in the hospital but also in the community. ${ }^{4}$ ESBL-E have been reported to take over the leading role as causative agents of neonatal infections that result in increased neonatal morbidity and mortality. ${ }^{5}$ Several outbreaks in NICUs due to multidrugresistant Klebsiella pneumoniae have been reported recently., Even more dramatic, temporally separated case clusters of New Delhi metallo-beta-lactamase $\left(b / a_{\text {NDM }}\right)$ and carbapenemase producing K. pneumoniae have been observed in a NICU in Nepal. ${ }^{8}$ The emergence of these transmissible carbapenemases makes it particularly essential to understand and subsequently prevent the transmission of multidrug-resistant bacteria on neonatal wards.

Colonization of the neonatal gut begins at, and most likely even before, the time of birth. This is a crucial process as beneficial functions of the commensal bacteria may be particularly important in the immature intestine of newborns. Bacteria that first colonize the neonatal gut are from different sources. This includes the maternal microbiota from skin, vagina, rectum, but also bacteria from the neonatal ward in case of hospitalization. ${ }^{9}$ Acquisition of maternal flora by neonates can occur during labor and through direct (hands and kangorooing) and indirect contacts (for example, breast milk) after birth. Transmission of ESBL-E on neonatal wards is suspected to be a product of import into and spread within the NICU. ${ }^{10}$

In this month's issue of the Journal, Rettedal et al. ${ }^{11}$ highlight the fact that maternal-neonatal transmission constitutes a substantial risk for colonization of infants with ESBL-E. Even though the prevalence of ESBL-E carriage among pregnant women in this Norwegian cohort is low (2.9\%), the maternal-neonatal transmission rate turned out to be $36 \%$. Recently, we reported the prevalence of ESBL-E among mothers of very low birth weight (VLBW, $<1500 \mathrm{~g}$ ) infants to be $11.1 \%$ (15 of 135) in a German NICU. However, ESBL-E screening only included those mothers with expected complications of birth and thus a higher risk of colonization. In our study, we also identified the mother as most important risk factor for colonization with ESBL-E among VLBW $(<1500 \mathrm{~g})$ infants. $^{12}$

Continuous surveillance of neonates by weekly screening for multidrug-resistant bacteria is recommended for NICUs to decrease neonatal acquisition of resistant bacteria. ${ }^{10,13}$ Considering the findings mentioned above, in addition to screening of preterm infants, it also seems reasonable to screen their mothers for ESBL-E. These measures should be restricted to preterm infants and their mothers owing to their substantially higher risk to develop neonatal sepsis compared with term infants. ${ }^{14}$ However, the identification of the mother as colonized with ESBL-E and therefore a risk to their infants should not lead to any barriers between mother and child. The higher fatality rate of neonatal infections with ESBL-E is most likely due to inadequate antibiotic treatment. ${ }^{15}$ Therapeutic mismatch has been reported as the most important reason for complicated courses of infection, increased lengths of stay, infection-related mortality and neurodevelopmental delays. ${ }^{13}$ This, however, should not encourage an uncritical use of second- or third-line antibiotics (for example, carbapenems) in all neonates with suspected sepsis. ${ }^{13}$ Screening for maternal ESBL-E should rather lead to an early choice of appropriate antibiotic therapy in case of a systemic neonatal infection.

Rettedal and colleagues show that only 14 of 26 (53.9\%) pregnant women tested positive for ESBL-E at the 36th gestational week were still positive when admitted to the obstetrics for delivery. Thus, it seems to be most reasonable to screen mothers of preterm infants for ESBL-E during delivery. Subsequently, good communication between obstetrical wards and NICUs is required to transfer positive screening results.

\section{CONFLICT OF INTEREST}

The authors declare no conflict of interest.

\section{LA Denkel, P Gastmeier and B Piening Institute of Hygiene and Environmental Medicine, Charité Universitätsmedizin Berlin, Berlin, Germany E-mail: luisa.denkel@charite.de}

\section{REFERENCES}

1 Tzialla C, Borghesi A, Pozzi M, Stronati M. Neonatal infections due to multiresistant strains: epidemiology, current treatment, emerging therapeutic approaches and prevention. Clini Chim Acta 2015 (epub ahead of print 4 March 2015; doi:10.1016/j.cca.2015.02.038).

2 Schwab F, Zibell R, Piening B, Geffers C, Gastmeier P. Mortality due to bloodstream infections and necrotizing enterocolitis in very low birth weight infants. Pediatr Infect Dis J 2015; 34(3): 235-240.

3 Shah J, Jefferies AL, Yoon EW, Lee SK, Shah PS. Risk factors and outcomes of late-onset bacterial sepsis in preterm neonates born at $<32$ weeks' gestation. Am J Perinatol 2015; 32(7): 675-682.

4 Pitout JD, Nordmann P, Laupland KB, Poirel L. Emergence of Enterobacteriaceae producing extended-spectrum beta-lactamases (ESBLs) in the community. $J$ Antimicrob Chemother 2005; 56(1): 52-59.

5 Stoll BJ, Hansen N, Fanaroff AA, Wright LL, Carlo WA, Ehrenkranz RA et al. Changes in pathogens causing early-onset sepsis in very-low-birth-weight infants. N Engl J Med 2002; 347(4): 240-247.

6 Haller S, Eller C, Hermes J, Kaase M, Steglich M, Radonic A et al. What caused the outbreak of ESBL-producing Klebsiella pneumoniae in a neonatal intensive care unit, Germany 2009 to 2012? Reconstructing transmission with epidemiological analysis and whole-genome sequencing. BMJ Open 2015; 5(5): e007397.

7 Sumer S, Turk Dagi H, Findik D, Arslan U, Aktug Demir N, Ural O et al. Two outbreaks of ESBL-producing Klebsiella pneumoniae in a neonatal intensive care unit. Pediatr Int 2014; 56(2): 222-226.

8 Stoesser N, Giess A, Batty EM, Sheppard AE, Walker AS, Wilson DJ et al. Genome sequencing of an extended series of NDM-producing Klebsiella pneumoniae isolates from neonatal infections in a Nepali hospital characterizes the extent of community- versus hospital-associated transmission in an endemic setting. Antimicrob Agents Chemother 2014; 58(12): 7347-7357. 
9 Adlerberth I, Wold AE. Establishment of the gut microbiota in Western infants Acta Paediatr 2009; 98(2): 229-238.

10 Benenson S, Levin PD, Block C, Adler A, Ergaz Z, Peleg O et al. Continuous surveillance to reduce extended-spectrum beta-lactamase Klebsiella pneumoniae colonization in the neonatal intensive care unit. Neonatology 2013; 103(2): 155-160.

11 Rettedal S, Löhr IH, Bernhoff E, Natås O, Sundfjord A, Øymar K. Extended-spectrum beta-lactamase-producing Enterobacteriaceae among pregnant women in Norway; prevalence and maternal-neonatal transmission. J Perinatol 2015; 35(11): 907-912.

12 Denkel LA, Schwab F, Kola A, Leistner R, Garten L, von Weizsacker K et al. The mother as most important risk factor for colonization of very low birth weight (VLBW) infants with extended-spectrum beta-lactamaseproducing Enterobacteriaceae (ESBL-E). J Antimicrob Chemother 2014; 69(8): 2230-2237

13 Simon A, Tenenbaum T. Surveillance of multidrug-resistant Gram-negative pathogens in high-risk neonates—does it make a difference? Pediatr Infect Dis J 2013; 32(4): 407-409.

14 Polin RA, Denson S, Brady MT. Epidemiology and diagnosis of health careassociated infections in the NICU. Pediatrics 2012; 129(4): e1104-e1109.

15 Tsai MH, Chu SM, Hsu JF, Lien R, Huang HR, Chiang MC et al. Risk factors and outcomes for multidrug-resistant Gram-negative bacteremia in the NICU. Pediatrics 2014; 133(2): e322-e329. 